\title{
Safe Medication Swallowing in Dysphagia: A Collaborative Improvement Project
}

Lawrence D. Jackson, Jane Little, Edward Kung, Evelyn M. Williams, Krystyna Siemiatkowska and Suzanne Plowman

\begin{abstract}
Episodes of choking during medication administration to patients with dysphagia prompted a chart audit and caregiver interview to identify system problems that allowed inappropriate drug administration to occur. Sixty elderly patients residing on two patient care areas in a 500-bed complex continuing care facility were studied. The audit explored the actual nursing medication administration methods and compared this to the information obtained from various communication tools including instructions that appeared on the medication administration record (MAR), the current diet order, the recommendations of the speech-language pathologist (SLP) and comments on the nursing care plan. The audit yielded a number of discrepancies between nursing actions and the instructions obtain from these sources.
\end{abstract}

We proposed that changes to the process of communicating medication swallowing recommendations among team members would lead to greater patient safety. Major practice changes included the use of standardized language by the SLP when making recommendations, the writing of SLP recommendations in the doctor's orders, the inclusion of SLP recommendations on the MAR and the creation of a "dysphagia alert" on the pharmacy computer system. An educational intervention was conducted to implement process changes. Its effectiveness was evaluated using a pre- and post-test and a participant satisfaction survey. A post-implementation audit showed compliance with the practice change.

In summary, process changes were implemented to improve compliance with SLP medication-related swallowing recommendations and to prevent the inadvertent prescribing, dispensing or crushing of sustained-release medications in patients with dysphagia. 
atients with dysphagia (swallowing difficulty) are at risk when a modification of the medication dosage form is not appropriately carried out and are at risk for adverse drug events caused by the inappropriate modification of the dosage form through the crushing of a tablet or capsule that should not be crushed. This article describes the extent of inappropriate medication administration identified in a population of elderly patients with dysphagia, contributing factors and the steps taken to ensure appropriate administration.

Dysphagia occurs commonly among residents living in longterm care facilities (LTCFs) (O'Brien and Barrow 1991; Trupe and Siebens 1984) and has been generally shown to be higher in LTCFs (ranging from 31.0 to $65.9 \%$ ) than in the general community (16.6\% of individuals aged 87 and older) or in hospitals (12 to 13\%) (Bloem et al. 1990; Groher and Bukatman 1986; Layne et al. 1989; Smithard 1996; Trupe and Siebens 1984). Many LTCF residents have illnesses causing dysphagia, including neurological and neuromuscular diseases, structural changes in the oropharynx, salivary changes and psychological illness (Sonies 1992). Impaired swallowing can lead to a number of serious consequences, such as aspiration, upper airway obstruction, malnutrition, dehydration and increased mortality (Hudson et al. 2000; Palmer and Drennan 2000; Siebens et al. 1986). Therefore, the act of swallowing places the patient with dysphagia at risk. To ensure safety during oral medication administration, patients with dysphagia require an appropriate oral dosage form or modification of the dosage form. Nurses may also modify medications according to patient preference, even if there is no difficulty with swallowing food. However, certain modifications are undesirable, such as the crushing of enteric-coated or sustained-release tablets, and can lead to adverse events. Examples of such adverse events include opioid overdose occurring when sustained-release morphine products are crushed, abnormal movements occurring when sustainedrelease levodopa-carbidopa is crushed and the loss of efficacy of medications such as omeprazole, which are destroyed by stomach acid when crushed (Cornish 2005; Wright 2002a, 2002b).

Effective communication among healthcare professionals within the hospital is required to safeguard patients with dysphagia. Our aim was to promote appropriate drug administration in patients with dysphagia through improved communication among caregivers and to in turn reduce the likelihood of swallowing-related adverse events. Since it would be difficult to track actual swallowing-related adverse events, our primary outcome was adherence to process changes. This article describes the implementation and evaluation of process improvements designed to ensure appropriate and safe medication administration in patients with dysphagia.

\section{Description of the Problem}

Choking episodes and instances of inappropriate administra- tion of medications to dysphagic patients have been reported in our hospital sporadically but have not been systematically quantified, nor have individual events been correlated with specific patient outcomes. However, based on the premise that inappropriate drug administration in patients with dysphagia (i.e., the crushing of sustained-release or enteric-coated tablets or the administration of oral medications with water) is undesirable and may be associated with adverse events (e.g., choking episodes, adverse drug reactions resulting from the immediate release of drug from a sustained-release product, refusal to take medications), implementation of procedures that limit the chance of this occurring should improve patient safety. Rootcause analysis revealed that a lack of awareness of dysphagia, due to poor communication practices among the healthcare team members, contributed to inappropriate medication administration in these patients. A systems improvement was implemented to remedy the problem.

\section{Background and Rationale}

In our facility, the speech-language pathologist (SLP) assesses patients with dysphagia and recommends the most appropriate diet texture (food and liquid components) as well as any modifications to oral medications that are required to promote safe swallowing. Communication of this information occurs through a consult note in the chart. However, existing procedures provided no assurance that the pharmacist reads this note or that the nurse relays SLP recommendations regarding medication swallowing to the pharmacy. Because nurses use a pharmacy-generated medication administration record (MAR), they routinely requested that the pharmacy add messages to the "comments" section of the MAR related to swallowing or other concerns; however, this occurred inconsistently. Another potentially unsafe practice involved the switching of solid medications to a liquid form, prior to receiving specific SLP recommendations, for patients who developed acute dysphagia. This was deemed unsafe since the patient may have difficulty with both solids and liquids.

Due to the occurrence of medication swallowing-related problems and the inconsistency with which information was being relayed to the pharmacy, existing communication procedures between the nurse, pharmacist, physician and SLP were examined and new processes were designed and implemented to address the problem (Coles et al. 2005; Wreathall and Nemeth 2004).

\section{Methods}

Using a quality improvement framework (Institute for Healthcare Improvement 2007), we studied existing communication procedures used to convey medication swallowingrelated concerns among the healthcare team and the method used by nurses to administer oral medication in patients with 
dysphagia. The study sample included patients residing on two complex continuing care units of a 500-bed LTCF affiliated with a tertiary acute care hospital. We conducted a chart and MAR audit, interviewed the primary nurse for each patient and compared SLP recommendations with actual nursing practice, and examined the extent to which SLP recommendations appeared on various documents (Table 1).

For the purposes of this study, dysphagia was defined by the presence of a modified texture diet order on the patient's chart. Modified diet orders included various food textures (pureed, minced and chopped), three graduated levels of thickened liquids (nectar-thick, honey-thick and pudding-thick) and "no mixed consistencies" (foods containing both solid and liquid components combined, such as vegetable soup). It was anticipated that patients with these diet orders would require the nurse to modify the medication (e.g., crushing or halving tablets, mixing crushed or whole tablets in food or opening capsules and mixing the powder in food) prior to administration to facilitate safe swallowing. Patients receiving gastric tube feeding and designated nothing by mouth, or NPO, are considered to have dysphagia but are not routinely seen by the SLP and were not included in the analysis. Key components of the audit included the current diet order, the presence of SLP medication swallowing recommendations on the MAR and nursing Kardex and the presence of SLP medication swallowing recommendations on the chart (i.e., consult note). These documents represent the primary means of communicating medication administra-

Table 1. Initial and post-intervention audit

\begin{tabular}{|l|l|l|}
\hline Parameter & $\begin{array}{l}\text { Initial Audit, } \\
\text { August 2003 }\end{array}$ & $\begin{array}{l}\text { Post-intervention } \\
\text { Audit, June 2005 }\end{array}$ \\
\hline No. of patients & 60 & 63 \\
\hline Patients with dysphagia & $22(37 \%)$ & $25(40 \%)$ \\
\hline MAR comments exist & $15 / 22(68 \%)$ & $23 / 25(92 \%)$ \\
\hline SLP recommendations on chart & $12 / 22(55 \%)$ & $21 / 23(91 \%)$ \\
\hline SLP recommendations on Kardex* & $1 / 22(5 \%)$ & N/A \\
\hline MAR comment agrees with SLP recommendations & $8 / 12(66 \%)$ & $23 / 25(92 \%)$ \\
\hline Nursing interventiont agrees with MAR comments & $15 / 15(100 \%)$ & $21 / 23(91 \%)$ \\
\hline $\begin{array}{l}\text { Nursing interventiont agrees with SLP } \\
\text { recommendations }\end{array}$ & $8 / 12(66 \%)$ & $23 / 25(92 \%)$ \\
\hline Nursing interventiont agrees with the diet order & $10 / 22(45 \%)$ & $24 / 25(96 \%)$ \\
\hline Pharmacy computer has "dysphagia alert" & None & $100 \%$ \\
\hline
\end{tabular}

MAR comments = speech-language pathologist (SLP) recommendations entered into the "comments" section of the medication administration record (MAR); N/A = not applicable.

*Kardex = customary care document.

†Nursing intervention = any action to modify a medication to facilitate swallowing tion instructions among nurses and between the various stakeholders (i.e., nurse, pharmacist, physician and SLP).

The results of the audit were used to design a new communication process and stakeholder responsibilities. The implementation of process changes was supported by education sessions, which were evaluated using pre- and post-education test of knowledge. General dissemination to nursing staff throughout the hospital was conducted through an education campaign using teams composed of a nurse educator, pharmacist and SLP. Two years after the implementation, we conducted a follow-up audit on the original study units to evaluate the sustainability of the process change (see Table 1). Patient consent to participate was not obtained due to the quality improvement nature of this initiative and because the improved communication process was applied to all patients with dysphagia in the study group.

\section{The initial audit revealed an inefficient and ineffective communication process that resulted in inappropriate medication administration in a significant number of patients with dysphagia.}

\section{Results and Interpretation of the Initial Audit}

Results of the initial audit conducted in August 2003 are presented in Table 1. Although we anticipated that all patients with dysphagia would receive medications in a modified form, this occurred in only 15 of $22(68 \%)$ of the cases. The other patients with dysphasia received medications with water - a potentially unsafe practice. When the various communication documents were examined, SLP medication swallowing recommendations were present on the MAR for $68 \%$ and on the Kardex for 5\% of the patients with dysphagia. An SLP consult note was present on only $55 \%$ of the charts. Consult notes had been relocated to an archival chart in the remaining cases.

When information from the nursing interview regarding the actual medication administration method (nursing action) was compared with the various communication documents, the nurses' actions agreed with the MAR, Kardex and SLP consult note in $100 \%, 100 \%$ and $66 \%$ of the cases, respectively. When we examined the extent to which the nurses modified medication based upon whether the patient was receiving a modified texture diet, a $45 \%$ correlation was found. A review of 
SLP consult notes revealed that recommendations for swallowing solid oral medications were present, but recommendations regarding the administration of liquid medications were absent.

In summary, the initial audit revealed an inefficient and ineffective communication process that resulted in inappropriate medication administration in a significant number of patients with dysphagia. The documents that nurses relied on for guidance at the time of medication administration did not reliably contain SLP recommendations, and, in many instances, the recommendations had been removed from the current chart. The existence of a modified diet texture order alone did not ensure that medications would be modified prior to administration. However, we did observe that nursing actions consistently agreed with the MAR, a correlation that became central in our process redesign.

\section{Interventions}

\section{Process Redesign and} Stakeholder Responsibilities

The new process design is summarized in the Safe Medication Swallowing in Dysphagia algorithm (Figure 1 and Table 2). Changes included the placement of SLP swallowing recommendations in the comments section of the MAR (Figure 2). This ensures that SLP recommendations are available to the nurse at the time of medication administration. The SLPs agreed to write their medication swallowing recommendations for both solid and liquid medications using standard phrases. These are to be written on the doctor's order sheet in addition to the consult note. The pharmacy automatically receives all copies of the doctor's order sheet, making this a shared communication document that fulfills a key aspect of our process redesign. The pharmacists enter

\section{Table 2. Key process changes}

- SLP to use standard phrases to make medication-related swallowing recommendations

- SLP recommendations to be written on the doctor's order sheet (pharmacy automatically receives copies of the doctor's order sheet)

- SLP to include recommendations for both solid and liquid oral medications

- Pharmacist to enter SLP recommendations in "comments" section of MAR

- Pharmacist to enter a "dysphagia alert" in the pharmacy computer system

$M A R=$ medication administration record; $S L P=$ speech-language pathologist.

\section{Figure 1. Safe Medication Swallowing in Dysphagia}

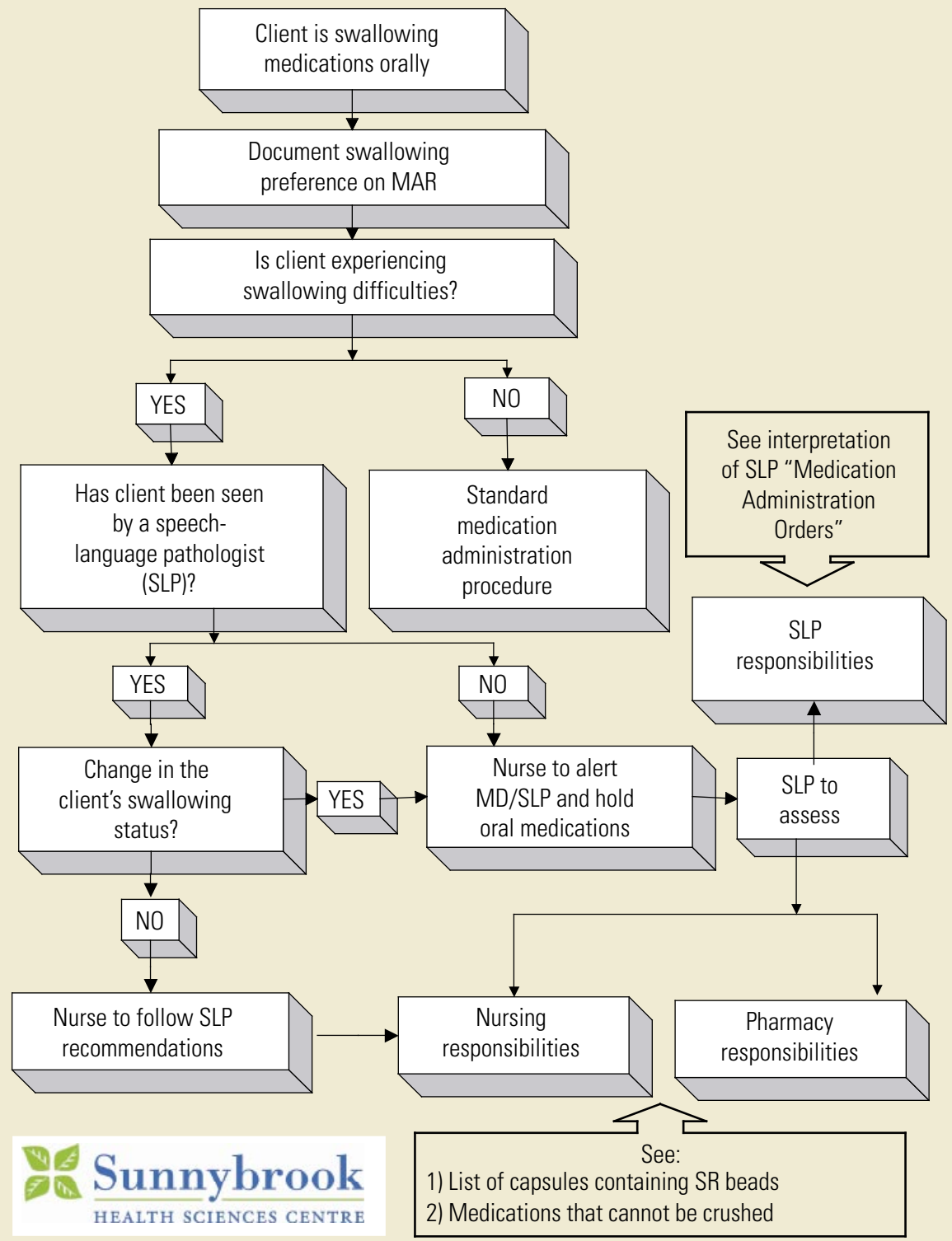

MAY 2004

MAR = medication administration record; $S R=$ sustained release .

Source: Reproduced with permission from Sunnybrook Health Sciences Centre. 
the SLP recommendations in the comments section of the MAR and flag the computer profile with a "dysphagia alert" (Figure 3). With this change, pharmacists entering medication orders must acknowledge the pop-up alert before proceeding. This feature enables the pharmacist to contact the physician promptly to discuss the most appropriate dosage form that the patient can safely swallow. The alert feature has been used previously for allergies, but this was its first application for dysphagia.
The nurse is responsible for monitoring patients closely during medication administration and notifying the physician and SLP if a change in swallowing status occurred.

\section{SLP Standard Phrases and Other Resources}

To avoid misinterpretation of SLP instructions, a common language, in the form of standard phrases, was created for use by the SLP when describing specific modifications to solid or liquid medications (Tables 3 and 4). Other resource materials included lists of medications that should not be crushed and medications available as sustained-release beads within a capsule, which can be opened but not crushed. All these documents were placed in the MAR binder for easy access.
Figure 3. Pharmacy computer system alert pop-up screen showing the dysphagia alert message

\section{Note Alert}

Crush tablets/open capsules and give with puree. Mix liquid medications in food.

\section{Education and Process Spread}

The process changes and stakeholder responsibilities were disseminated through a formal education campaign delivered during staff meetings of the respective stakeholders (Table 5). Dissemination to nursing staff was conducted via unit in-services and the posting of supporting documents on units and on the hospital intranet. The effectiveness of the initial nursing education sessions was evaluated using a written pre- and post-education knowledge test. (The test is available from the authors.) The test examined knowledge of diet textures, types of dosage forms that should not be crushed and stakeholder responsibilities. Participants were asked to complete a feedback form to determine satisfaction with these education sessions. The average knowledge score of $60 \%$ improved to $80 \%$ after the in-service. Average satisfaction score for the educational sessions was 4.5 out of 5 .

"Exact wording of standard phrases appears in the "comments" section on the MAR. TPureed food = applesauce, yogurt, pudding or other pureed food. When mixing in pureed food, put medication in a small portion of food (in case patient does not consume all of the mixture.) 
Table 4. Medication administration orders for liquid medications in dysphagia

\begin{tabular}{|l|l|l|}
\hline Standard Phrases* & Typical Diet Order & Interpretation of Standard Phrases \\
\hline No liquid medication & Pudding-thick liquids & Requires very thick liquids \\
\hline Mix liquid medication in food & Nectar-thick liquids, honey-thick liquids & Does not require very thick liquids \\
\hline Liquid medication allowed & $\begin{array}{l}\text { All diets except nectar-thick liquids, honey- } \\
\text { thick liquids and pudding-thick liquids }\end{array}$ & Can tolerate any volume of liquid \\
\hline Medication must be in liquid form & Clear fluids, wired jaw diet & Can swallow liquids but not solid food \\
\hline
\end{tabular}

*Exact wording of standard phrases appears in the "comments" section on the MAR.

Table 5. Education program for nurses

\section{Speech-language pathologist presentation:}

- Swallowing physiology and pathophysiology

- Risks due to dysphagia

- Diet texture, consistency of solids and liquids

- Standard order phrases and their interpretation

\section{Stakeholder responsibilities:}

- Nurse, SLP, pharmacist, physician

\section{Documents:}

- Medications which cannot be crushed and rationale

- Medications available as sustained-release beads

\section{Evaluation:}

- Pre- and post-education knowledge test

- Participant evaluation and feedback on the education session

The new process was implemented on the two study units first and then spread to all units of the LCTF. Spread to the acute care hospital occurred through a series of information meetings with stakeholders, specifically acute care SLPs, pharmacists and nursing leaders.

\section{The average knowledge score of $60 \%$ improved to $80 \%$ after the in-service.}

\section{Analysis and Interpretation of the Post-intervention Audit}

In June 2005, a post-intervention audit on the two original study units revealed over $90 \%$ adherence with the process changes that were implemented in 2003 (see Table 1), which is our primary outcome. We anticipate that the process changes will result in a reduction in swallowing-related adverse events. In the two cases where nursing action did not adhere to the SLP recommendations, patients had a "no mixed consistencies" restriction. This restriction implies mild dysphagia, and the nurse or patient may have felt that receiving medications whole with water was safe. SLP recommendations had been moved to the archival chart in two cases. In the two cases where nursing action disagreed with the MAR, it was due to patient preference. It is clear that nurses generally continued to follow the MAR instructions closely. Entering the SLP recommendations on the MAR was a key factor in improving nursing adherence with SLP recommendations from $66 \%$ in the initial audit to $93 \%$ in the second audit. There was complete adherence with the pharmacy computer alert.

\section{Entering the SLP recommendations on} the MAR was a key factor in improving nursing adherence.

\section{Summary}

Patient safety is now prominent on the agenda of healthcare institutions (Baker et al. 2004). Understanding the contributing factors leading to adverse events is seen as important for hospitals to correct deficiencies that might act as barriers to providing safe patient care (El-Jardali and Lagacé 2005). Inadequate communication of important information for the safe administration of medication to patients with swallowing difficulty was identified as the key contributing factor to adverse patient outcomes related to medication administration, such as choking and adverse events resulting from the inappropriate crushing of sustained-release tablets. Communication of SLP medication swallowing recommendations to nurses and pharmacists via the doctor's order sheet using standard order phrases was implemented as a simple change to existing procedures that remedied this communication gap.

Improved compliance with interdisciplinary communication procedures and medication administration practices was demonstrated in this study. The ease with which the process changes were implemented was gratifying and due, in part, to the fact that buy-in was required from a small number of staff in two of the stakeholder groups (i.e., pharmacists and SLPs). The larger nursing group had already demonstrated adherence with the MAR. The formal education campaign was considered important in raising the level of knowledge among the nursing staff regarding swallowing problems and associ- 
ated risks. Incorporating the pharmacy computer system and pharmacy-generated MAR in this process change was important in terms of sustaining the process, ensuring the communication loop between the SLP, pharmacist and nurse was closed and preventing the crushing of sustained-release medications. The "dysphagia alert" concept will be included in the physician computer order entry system that is being developed for our hospital. HQ

\section{About the Authors \\ Lawrence D. Jackson, RPh, BScPhm, is pharmacy clinical coordinator in Aging and Veterans Care (A\&VC), Sunnybrook Health Sciences Centre, Toronto, Ontario. He can be contacted at Lawrence.Jackson@sunnybrook.ca.}

Jane Little, MA, Reg CASLPO, SLP(C), was a speech-language pathologist in A\&VC as well as professional leader for speechlanguage pathology at Sunnybrook Health Sciences Centre when this study was conducted.

Edward Kung, RPh, BScPhm, is pharmacy supervisor in A\&VC, Sunnybrook Health Sciences Centre.

Evelyn M. Williams, MD, MHSc, CHE, FCFP, is assistant professor, Department of Family and Community Medicine, University of Toronto, Toronto, and head, Division of Long-Term Care, A\&VC, Sunnybrook Health Sciences Centre.

Krystyna Siemiatkowska, RN, was a staff nurse on the K2C and K2E Patient Care Units, Sunnybrook Health Sciences Centre, when this study was conducted.

Suzanne Plowman, RN, BA, is the patient care manager for the K2C and K2E Patient Care Units, A\&VC, Sunnybrook Health Sciences Centre.

\section{Acknowledgements}

Special thanks to the nurses of the $\mathrm{K} 2 \mathrm{C} / \mathrm{E}$ Patient Care Units and Lawrence Holley, RN, MN(c), for their contributions to this study. Thank you to Dr. Edward Etchells, director, Patient Safety Service, and staff physician, Division of General Internal Medicine, Sunnybrook Health Sciences Centre; and associate professor, Department of Medicine, University of Toronto, for his helpful comments.

\section{References}

Baker, G.R., P.G. Norton, V. Flintoft, R. Blais, A. Brown, J. Cox, E. Etchells, W.A. Ghali, P. Hébert, S.R. Majumdar, M. O’Beirne, L. Palacios-Derflingher, R.J. Reid, S. Sheps and R. Tamblyn. 2004. "The Canadian Adverse Events Study: The Incidence of Adverse Events among Hospital Patients in Canada." Canadian Medical Association Journal 170: 1678-86.

Bloem, B.R., A.M. Lagaay, W. Van Beek, J. Haan, R.A. Roos and A.R. Wintzen. 1990. "Prevalence of Subjective Dysphagia in Community Residents Aged over 87.” BMJ 300: 721-2.

Coles, G., B. Fuller, K. Nordquist and A. Kongslie. 2005. "Using Failure Mode Effects and Criticality Analysis for High-Risk Processes at Three Community Hospitals." Joint Commission Journal on Quality and Patient Safety 31(3): 132-40.

Cornish, P. 2005. "Avoid the Crush: Hazards of Medication Administration in Patients with Dysphagia or a Feeding Tube." Canadian Medical Association Journal 172(7): 871-87.

El-Jardali, F. and M. Lagacé. 2005. "Making Hospital Care Safer and Better: The Structure-Process Connection Leading to Adverse Events." Healthcare Quarterly 8(2): 40-8.

Groher, M.E. and R. Bukatman. 1986. "The Prevalence of Swallowing Disorders in Two Teaching Hospitals." Dysphagia 1: 3-6.

Hudson, H.M., C.R. Daubert and R.J. Mills. 2000. "The Interdependency of Protein-Energy Malnutrition, Aging, and Dysphagia." Dysphagia 15: 31-8.

Institute for Healthcare Improvement. 2007. Quality Improvement Model. Cambridge, MA: Author. <http://www.ihi.org/IHI/Topics/ Improvement/>. Retrieved December 9, 2007.

Laffel, G. and M. Blumenthal. 1989. "The Case for Using Industrial Quality Management Science in Health Care Organizations." Journal of the American Medical Association 262: 2869-73.

Layne, K.A., D.S. Losinski, P.M. Zenner and J.A. Ament. 1989. "Using the Fleming Index of Dysphagia to Establish Prevalence." Dysphagia 4: $39-42$.

O’Brien, P.J. and D. Barrow. 1991. "Prevalence of Eating Problems of Nursing Home Residents." Australian Journal of Human Communication Disorders 19(1): 35-43.

Palmer, J.B. and J.C. Drennan. 2000. "Evaluation and Treatment for Swallowing Impairments." American Family Physician 61: 2453-62.

Siebens, H., E. Trupe, A. Siebens, F. Cook, S. Anshen, R. Hanauer and G. Oster. 1986. "Correlates and Consequences of Eating Dependency in Institutionalized Elderly." Journal of the American Geriatrics Society 34: 192-8.

Smithard, D.G. 1996. "Feeding and Swallowing Problems in the Institutionalized Elderly." Clinical Rehabilitation 10: 153-4.

Sonies, B. 1992. "Oropharyngeal Dysphagia in the Elderly." Clinics in Geriatric Medicine 8(3): 569-77.

Trupe, E.H. and A.A. Siebens. 1984. "Prevalence of Feeding and Swallowing Disorders in a Nursing Home." Archives of Physical Rehabilitation 65: 651-2.

Wreathall, J. and C. Nemeth. 2004. "Assessing Risk: The Role of Probabilistic Risk Assessment (PRA) in Patient Safety Improvement." Quality and Safety in Health Care 13: 206-12.

Wright, D. 2002a. "Medication Administration in Nursing Homes." Nursing Standard 16(42): 33-8.

Wright, D. 2002b. "Swallowing Difficulties Protocol: Medication Administration.” Nursing Standard 17(14-15): 43-5. 